\title{
Modelling Frontier Mortality Using Bayesian Generalised Additive Models
}

\author{
Jason Hilton ${ }^{1}$, Erengul Dodd ${ }^{2}$, Jonathan J. Forster ${ }^{3}$, and Peter W.F. Smith ${ }^{1}$
}

\begin{abstract}
Mortality rates differ across countries and years, and the country with the lowest observed mortality has changed over time. However, the classic Science paper by Oeppen and Vaupel (2002) identified a persistent linear trend over time in maximum national life expectancy. In this article, we look to exploit similar regularities in age-specific mortality by considering for any given year a hypothetical mortality 'frontier', which we define as the lower limit of the force of mortality at each age across all countries. Change in this frontier reflects incremental advances across the wide range of social, institutional and scientific dimensions that influence mortality. We jointly estimate frontier mortality as well as mortality rates for individual countries. Generalised additive models are used to estimate a smooth set of baseline frontier mortality rates and mortality improvements, and country-level mortality is modelled as a set of smooth, positive deviations from this, forcing the mortality estimates for individual countries to lie above the frontier. This model is fitted to data for a selection of countries from the Human Mortality Database (2019). The efficacy of the model in forecasting over a tenyear horizon is compared to a similar model fitted to each country separately.
\end{abstract}

Key words: Mortality; demography; Bayesian methods; population forecasting.

\section{Introduction}

Modelling and forecasting mortality is a vital function for government bodies that produce official statistics. Population projections and life expectancy calculations depend on their production, and in turn these influence policy on public pensions, health spending, and planning. Official projections may gain from utilising data from across a range of countries (see, for example Raftery et al. 2013), as this greater depth of mortality experience may reveal the long-term pattern in mortality more clearly than any single country alone. Frontier (or 'best-practice') life expectancy, defined as the highest value of national life expectancy globally, has shown sustained increases over many decades (Oeppen and Vaupel 2002), and furthermore national life expectancies in different states appear to be converging (Wilson 2001). The extent to which we can expect these trends to continue in the long term is subject to debate (Olshansky et al. 2001; Vallin and Mesle 2009; Lee 2019). However, as highlighted by Oeppen and Vaupel (2002), previous predicted limits to life expectancy have been surpassed not long after they were proposed.

\footnotetext{
${ }^{1}$ Department of Social Statistics and Demography, University of Southampton, Highfield, Southampton, SO17 1BJ, United Kingdom. Emails: J.D.Hilton@soton.ac.uk, and P.W.Smith@soton.ac.uk

${ }^{2}$ School of Mathematical Sciences, University of Southampton, Highfield, Southampton, SO17 1BJ, United Kingdom. Email: E.Dodd@soton.ac.uk

${ }^{3}$ Department of Statistics, University of Warwick, Coventry, CV4 7AL, United Kingdom. Email: J.J.Forster@warwick. ac.uk
} 
The regularities in period life expectancy identified by Oeppen and Vaupel (2002) have obvious utility for forecasting, and a number of authors have taken up the challenge of producing forecasts based on extending these observed patterns in life expectancy into the future (Bijak et al. 2007; Torri and Vaupel 2012; Pascariu et al. 2018). However, as Lee $(2019,70)$ notes, period life expectancy is "a very particular and non-linear summary measure" based on the hypothetical experiences of a synthetic cohort, and the underlying age-specific rates appear to be a more fundamental quantity in the study of human mortality. The importance of the age-specific force of mortality is underlined by its role in evolutionary arguments about the ageing process (e.g., Wachter 1997; Wachter et al. 2014). Furthermore, in order to produce population projections, which are often the main goal of any demographic projection exercise, age-specific rates are needed in any case. Thus, forecasts based on regularities in life expectancy must also provide some method of decomposing this summary into age-specific mortality, hopefully in a way that captures the diversity of patterns in age-specific change in mortality across countries. For these reasons, we prefer to model log-mortality rates directly.

Oeppen and Vaupel (2002) make it clear that, initially at least, they do not see a contradiction between regularities in life-expectancy and in age-specific mortality (as Lee (2019) also points out). In arguing against an imminent limit to life expectancy, they cite papers by Lee and Carter (1992) and Tuljapurkar et al. (2000) that focus on an observed stability in improvements in log-mortality as evidence of a steady long-run stream of improvements. Furthermore, in the supplemental material to their article, they explicitly state that: "steady rates of change in mortality levels produce steady absolute increases in life expectancy: This relationship may underlie the linear trend of record life expectancy". This reasoning is based on analytical results going back to Keyfitz (1977) that show this relationship holds under the assumption of rates of mortality improvement that are constant with respect to age (Vaupel 1986; Vaupel and Canudas Romo 2000).

However, as Vaupel and Canudas Romo (2003) show, the time-derivative of lifeexpectancy is a weighted sum of rates of mortality improvements over age, the weights for which depend on the current level of mortality. As Aburto et al. (2020) illustrate, these weights shift to place a greater emphasis on older ages over time, so that at current Swedish levels of age-specific mortality, life-expectancy increases are mostly dependent on rates of mortality improvement at 70 and older, whereas in the past, infant mortality and mortality in middle ages were much more significant.

Historically, rates of mortality improvement have tended to be slower at older ages, so this shift towards an increased importance of old-age mortality may result in decelerating growth in life-expectancy. Interestingly, Lee (2019) identifies such a deceleration in the original series provided in Oeppen and Vaupel (2002), although only to a relatively small degree. In practice, the difference between linear lifeexpectancy growth and constancy in log-mortality improvements appears to be relatively slight. For instance, Figure 2 in Tuljapurkar et al. (2000) provides projections of life expectancy at birth derived from mortality forecasts using the model of Lee and Carter $(1992,791)$, which assumes linear mortality improvements at each age-specific rate. The median paths of these life expectancy forecasts are close to linear for the G7 countries. 


\section{The Mortality Frontier}

The model presented in this article relies on the concept of a mortality frontier; a schedule of mortality rates that represents the best achievable outcome by a national population at a given point in time, as determined by existing constraints provided by technologies, social and political norms, economic factors and population histories. Such a concept is necessarily hypothetical, in that one can always imagine ways in which such a limit could be breached. However, this idea of 'best-practice' mortality is widespread in the literature on mortality (Oeppen and Vaupel 2002; Torri and Vaupel 2012; Pascariu et al. 2018; Alho 2019), and our usage in this article differs only in that we apply it to underlying logmortality rates and not to life expectancy.

To make this concept more concrete, we consider the frontier as a mortality surface that is lower than, but as close as possible to, the force of mortality for all national populations of a reasonable size. As the force of mortality is an unobservable quantity, any attempt to estimate this frontier will be imperfect, but we show that such a concept may have utility for the purposes of forecasting. Although in common with other authors (e.g., Vallin and Mesle 2009) we focus on relatively large national populations to identify this mortality frontier, there is nothing fundamental about this level of analysis in the study of mortality. Small-scale subdivisions of populations would no doubt result in frontiers exhibiting lower mortality, to the extent that factors that might determine mortality differ between these sub-divisions.

This effect was noted by Vallin and Mesle (2009) and Bengtsson (2019), who point out that low historical mortality in New Zealand is likely in part due to a small population subject to positive selection via the process of migration. However, national populations are the primary focus of mortality modelling for official statistics agencies, so we focus on this level for pragmatic reasons.

A number of explanations exist as to why consistent declines in the hypothetical mortality frontier (whether defined at the level of mortality rates or life expectancy) occur. Oeppen and Vaupel (2002, 1029) describe a "regular stream of continuing progress" resulting from an "intricate interplay of advances in income, salubrity, nutrition, eduction, sanitation, and medicine”. In the supplemental material to their article, they highlight that as mortality at younger ages drops, scientific and governmental attention and the resources brought by continued economic growth can be focussed on maintaining progress at older ages. Oeppen (2019) expands on this theme with reference to a model that seeks to describe the relationship between national income and life expectancy at each time point through a technology function that describes the 'price' of a given level of mortality, deviation from which is determined by the particularities of the history and institutions of specific countries. Bengtsson (2019) also highlights that as with technological progress in economics, we might expect a penalty for innovators in terms of future progress, as they are unable to borrow ideas from more advanced neighbours. He notes that a repeated pattern, whereby particular countries accelerate to take the lead but subsequently slow down, could result in a long-run linear frontier trend.

Many authors highlight that the exact reason as to why mortality improvements should be linear is uncertain (Vallin and Mesle 2009; Lee 2019; Bengtsson 2019). However, it seems that there are enough potential explanations for us to seek to employ consistent regularities in frontier mortality in the pursuit of better projections. 
This article employs the Bayesian generalised additive mortality model of Hilton et al. (2019) to estimate frontier mortality rates and project them forward at the long-run rate of log-mortality improvement, modelling individual country mortality schedules as deviations from this frontier experience. Such an approach appears to be at odds with some recent work in the literature, which is concerned that forecasts based on the assumption of constant log-improvement systematically under-predict life-expectancy (Bohk-Ewald and Rau 2017; Bergeron-Boucher et al. 2017, 2018). This is not necessarily the case, as our model only assumes this regularity for the frontier. As individual countries 'catch up' with the mortality frontier, accelerations in their rate of mortality improvement are expected (Bengtsson 2019). The next section examines empirical evidence for linear declines in frontier log-mortality.

\section{Descriptive Analysis}

In order for such an approach to be suitable for mortality forecasting, we hope that frontier mortality does display the expected regularities. The Human Mortality Database provides a collection of mortality data spanning a wide range of developed countries, which are collectively ideal for examining the behaviour of the mortality frontier (Human Mortality Database 2019). Mortality data are typically defined in terms of the central mortality rate

$$
m_{x t}=\frac{D_{x t}}{R_{x t}},
$$

where $D_{x t}$ denotes the number of deaths of individuals aged between $x$ and $x+1$ during year $t$, and $R_{x t}$ is the exposure to risk during of the same group over that period, measured in terms of person-years lived. Ages may range from 0 to some maximum age $X$, with the latest year denoted by $T$. We define the empirical 'frontier' as the best (lowest) mortality rate at each year and age among all countries for which data are available:

$$
m_{x t}^{*}=\min _{c}\left(m_{x t c}\right),
$$

where $c$ indicates a particular country. Figure 1 plots the natural logarithm of empirical frontier mortality for females at forty-year intervals from 1816 to the present day. We can see from this plot that log-mortality appears to have declined more quickly over the last century than over the preceding 100 years, and furthermore, the rate of decline varies for different ages. Additionally, we can see that empirical frontier log-mortality is not smooth; considerable variability is observed for the youngest ages in particular, where death counts are low and random variability plays a greater role. Any proposed frontier model should be able to take into account these features of the data.

The pattern of mortality frontier improvement factors is an important consideration for modelling. Restricting ourselves to more recent years, we can observe the pattern of decline in empirical frontier mortality over time for particular ages in Figure 2. According to a quick observation, it would appear that such declines have been relatively linear since 1960.

Also of interest is the extent to which different countries contribute to the mortality frontier. Figure 3 plots tallies for each country of the number of individual age-specific mortality rate observations that form part of the empirical mortality frontier. The 


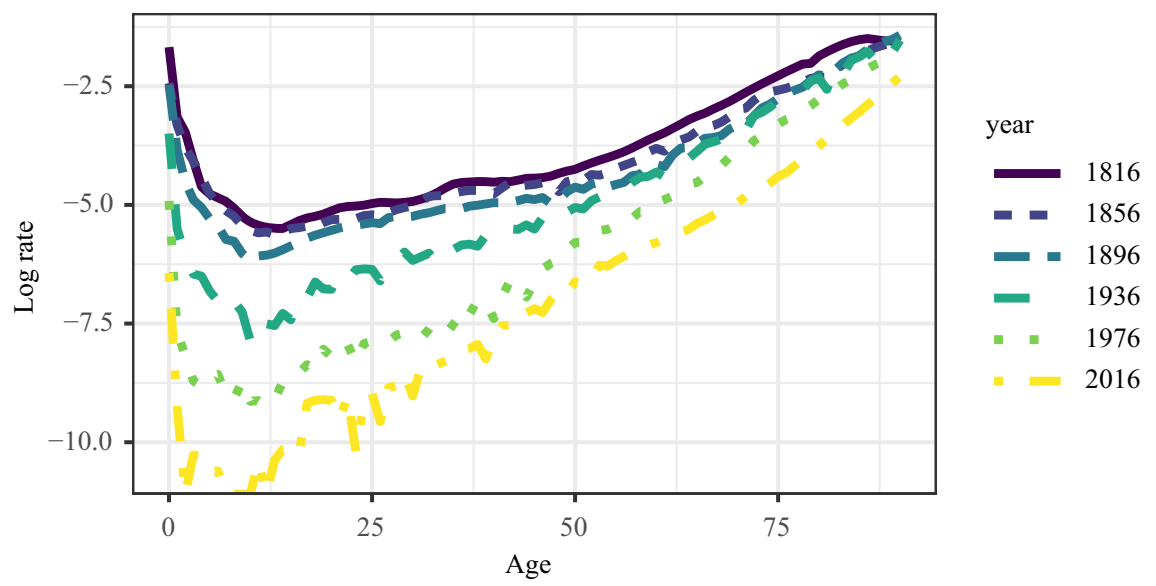

Fig. 1. Empirical frontier mortality for females in selected years. Human Mortality Database.

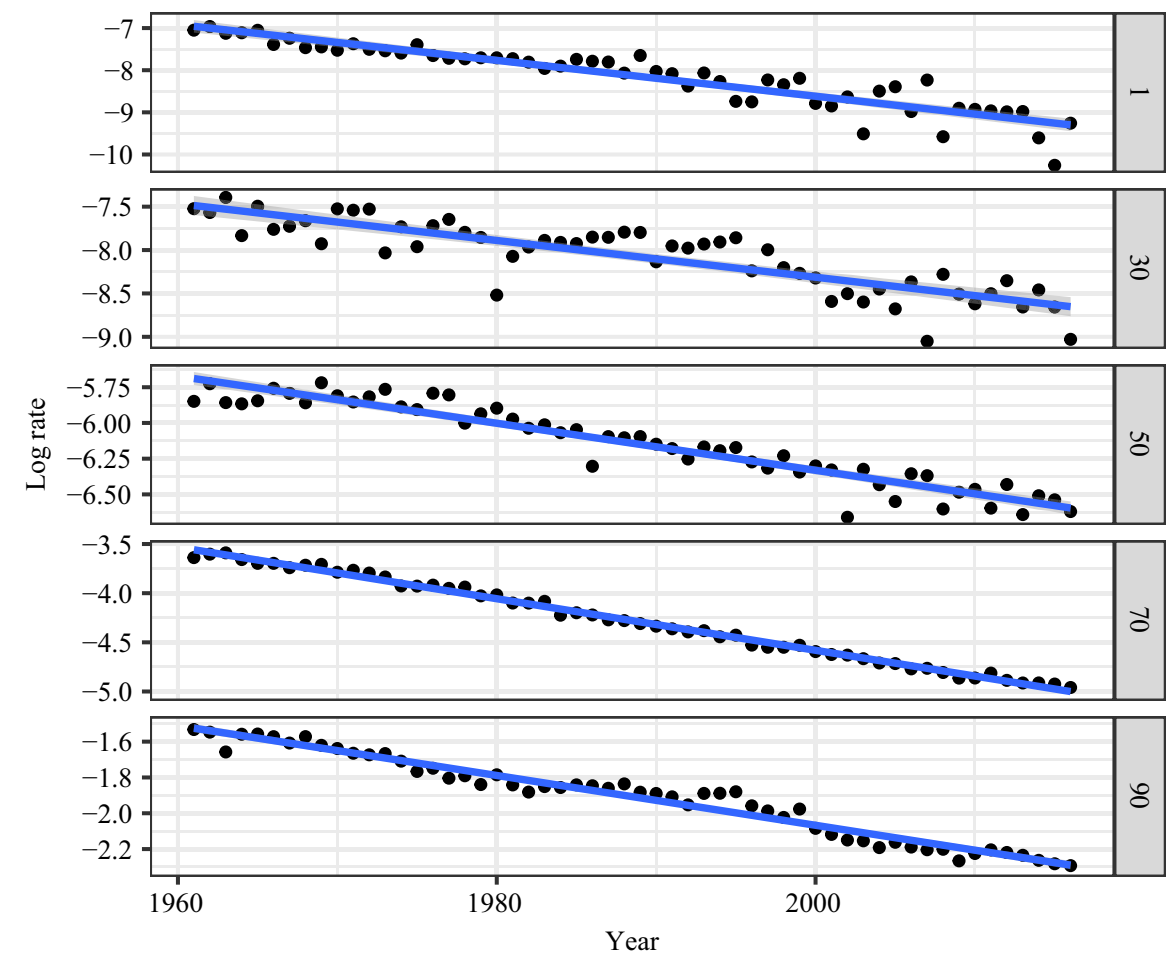

Fig. 2. Patterns of empirical frontier log-mortality Improvement by age. Human Mortality Database.

breakdown of these observations by age-group are also recorded. It is clear that although Japan and Norway are the biggest contributors, the frontier is not primarily made up of observations from one country. This suggests that we may be able to make gains in estimating the frontier with a model that uses information from multiple countries. Some of the countries identified as contributing to the frontier may seem surprising. For instance, 


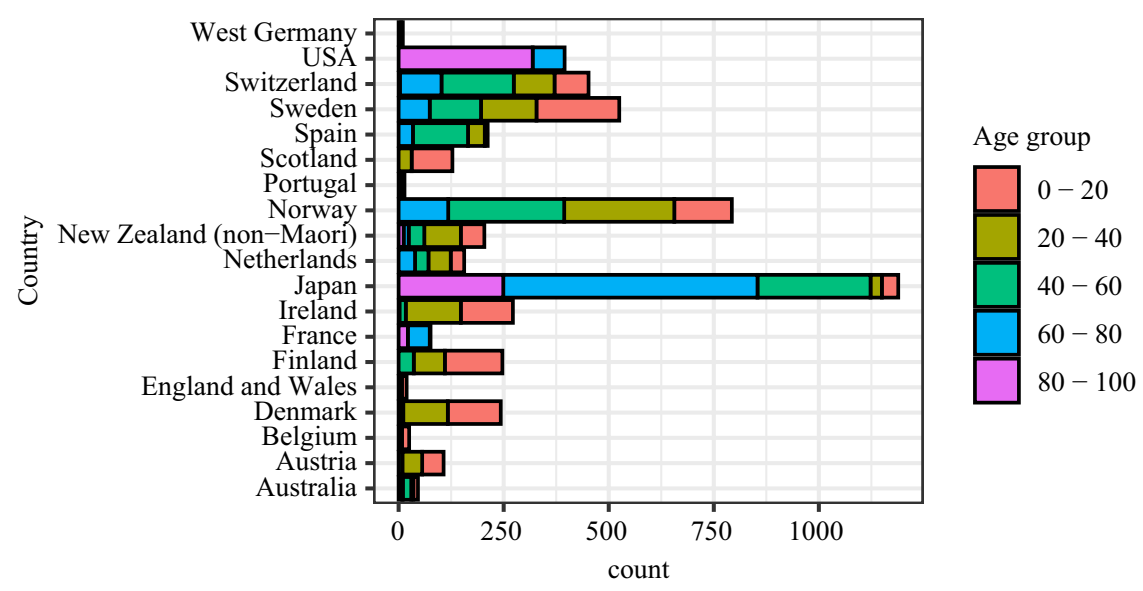

Fig. 3. Counts of country contributions to empirical frontier mortality by age group. Human Mortality Database.

Scotland contributes many observations to the frontier between ages 0 and 40, while England contributes very few, despite the latter having higher life expectancy over the period considered. This is because Scotland's much smaller population means that random variation is more likely to result in observed mortality rates that are very low, even if on average, rates are higher than for many of its peers. This also may explain the prominent contributions of other smaller countries such as Ireland, Finland and New Zealand to frontier mortality at young ages. This observation provides more motivation for a modelbased approach to estimating frontier mortality; simply using the best observed rate for each year and age will cloud our understanding of the long-term structural changes associated with declining frontier mortality. We can therefore make a distinction between the empirical mortality frontier and a hypothetical frontier that we wish to model.

The extent to which levels of frontier mortality improvement have persisted over time is also worth examining. Mortality improvement is typically measured using log mortality ratios (or improvement factors), defined as $\log \left(\frac{m_{x, t}}{m_{x, t-1}}\right)=\log \left(m_{x, t}\right)-\log \left(m_{x, t-1}\right)$. Figure 4 displays

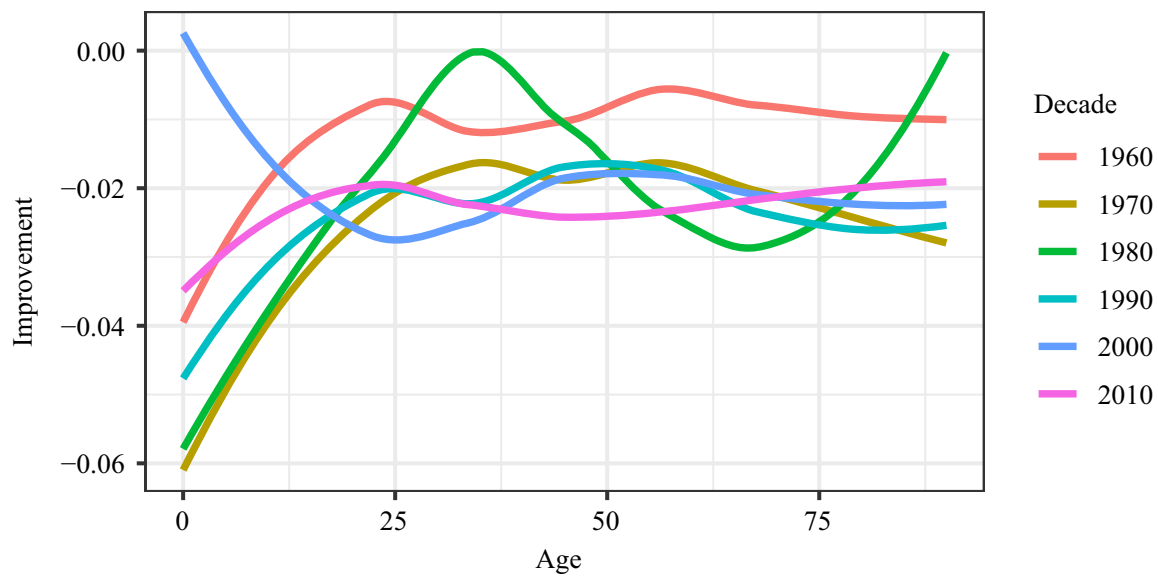

Fig. 4. Smoothed frontier mortality improvements by decade. Human Mortality Database. 
the average age-specific log mortality improvement ratios base on linear models fitted to data for each decade since 1960, smoothed using local weighted polynomial regression. While ages below 30 display low counts and are more likely to be subject to noise, ages from 40 onward are concentrated in a band around -0.02, particularly in the period since 1970 .

This empirical evidence supports the suggestion that there may be some utility in modelling and forecasting mortality with reference to the frontier defined for logmortality. Model-based techniques can help us better extract a representation of the mortality frontier from the empirical noise of detailed and varied cross-country data. The next section examines existing approaches to mortality modelling, with particular focus on models that borrow strength across countries and that involve 'best-practice' mortality.

\section{Models of Mortality}

There are various different approaches to the modelling of mortality, of which Booth and Tickle (2008) provides an extensive review. Mortality is the demographic component most amenable to forecasting; unlike migration and fertility, both the age pattern of the rates and the direction of change has remained steady over a very long time horizon. A few key approaches to mortality forecasting are highlighted in this section. One strand of the literature is based on the idea of reducing the dimensionality of the problem by identifying leading principle components of the matrix of log-mortality rates and using these for forecasting. The seminal paper in this area is Lee and Carter (1992). Their method decomposes the centred log-mortality rates into a time index describing the overall rate of mortality decline and a vector of age-specific factors describing the rate of decline of each age-specific rate relative to this index, so that $\log \left(m_{x t}\right)=a_{x}+b_{x} k_{t}$. The vectors $\boldsymbol{b}=$ $\left(b_{0}, b_{1}, \ldots, b_{X}\right)$ and $k=\left(k_{1}, k_{2}, \ldots, k_{T}\right)$ correspond to the first principal component of the centred log-rate matrix, and can therefore be estimated using singular value decomposition. Since only the index $k_{t}$ varies over time, the forecasting problem is much simplified. Typically, simple time series models suffice for $k_{t}$, and in particular the random walk with drift has been found to perform well. A wide of extensions of the LeeCarter model have been proposed, a testament to the simplicity and efficacy of the model (e.g., Lee and Tuljapurkar 1994; Booth et al. 2006; Li et al. 2013). Hyndman and Ullah (2007) provide an extension of the Lee-Carter model from within the functional data analysis framework, allowing for more than one principal component to be employed in forecasting, and for the smoothing of the age-profile of mortality decline.

From a different perspective, Currie et al. (2004) employ two-dimensional penalised Bsplines to capture log-mortality rates, allowing considerable flexibility in the shape of the mortality surface. Forecasting is possible through the interpretation of the smoothing penalisation of basis function coefficients as a time series model, allowing basis function coefficients for new periods to be generated. Also employing penalised B-splines, Hilton et al. (2019) fit generalised additive models in order to capture smooth age, age-specific improvement, and cohort components together with a period effect capturing deviations from the linear trend (for which roughness is deemed appropriate). Taking a more general view, Cairns et al. (2009) describe a family of models in which log-mortality is considered as a sum of terms of age, period and cohort effects, possibly including interactions. This family includes the Lee-Carter model and the model of Currie et al. (2004) as special cases. 


\subsection{Coherent Models}

Many researchers have attempt to utilise information from multiple countries or populations to produce better forecasts. This often arises within the context of attempting to ensure coherence between male and female forecasts, or between mortality forecasts across many countries. Models that forecast separate populations with constant rates of mortality improvement can expect to see predictions diverge in the future, to an extent which is unsupported in the data (Hyndman et al. 2013). Similarly, given that the gap between male and female life expectancy is narrowing in many countries, separate longterm forecasts by sex are likely to show a crossover in mortality rates. This seems similarly implausible given evidence that there may be some biological basis for difference in male and female mortality and ageing (Luy 2003). By identifying common trends across populations and allowing individual populations to converge towards such trends, coherence is ensured. While the trends involved do not refer to the mortality frontier discussed above, there are many commonalities between the coherent mortality models and the approach proposed in this article.

Several authors have attempted to produce models that avoid such incoherent forecasts. Li and Lee (2005) fit the Lee-Carter model to all-country mortality, and specify additional mean-reverting bi-variate terms that capture divergences from this central trend. Kleinow (2015) develop this work to include different populations with the same Lee-Carter age term $\beta_{x}$ but different time terms. Bergeron-Boucher et al. (2017) also adapt the model of Li and Lee (2005) to apply to their compositional data (CODA) mortality modelling framework, where the modelling target is the distribution of life table deaths rather than the mortality rates themselves. Still working with a principle component framework, Hyndman et al. (2013) extend the model of Hyndman and Ullah (2007) to target the product and ratio of sub-population mortality rates, modelling these transformations using functional principal component time series techniques, and taking advantage of the fact that these products and ratios are uncorrelated, making uncertainty quantification for forecasts more straightforward. They find that coherent forecasts improve overall accuracy in comparison to independently fitted equivalents. Adapting existing models to provide for coherence is a common approach: Biatat and Currie (2010) provide an extension of the model of Currie et al. (2004) that allows mortality for two populations to be modelled; the first using the original model, and the second as the first population plus a gap, comprising the sum of two one-dimensional splines, one aligned along the age axis and other against time. Cairns et al. (2011) also consider a two-population model, but using a simple AgePeriod-Cohort model as a test case. They describe and implement various ways of enforcing coherence in the evolution of period and cohort effects, including cases where one population is dominant. Enchev et al. (2017) discuss and evaluate a range of different multi-population models, including the Li and Lee (2005) model, and find that both the common age effect model of Kleinow (2015) and the Li and Lee (2005) model produce satisfactory, albeit different, forecasts.

The hierarchical model of mortality feeding into the United Nations World Population Projections provide an elegant way of ensuring coherence in mortality forecast across the globe, while also allowing forecasts to be made for countries with incomplete data (Raftery et al. 2013). The time evolution of life expectancy for each country is modelled 
using a stochastic double logistic function, with the parameters drawn from a global distribution. Such a model also allows for missing data, an important problem when modelling mortality in developing countries. Bohk-Ewald and Rau (2017) similarly adopt a hierarchical Bayesian perspective, but allow age-specific mortality improvements to depend on time in a linear or exponential fashion, and assume the rates of change and intercepts of such models are drawn from common global distributions. The extent to which sub-population forecasts borrow strength can also be specified; Schinzinger et al. (2016) provide a family of mortality forecasting models deriving from the Lee-Carter specification, but with mortality improvements rather than mortality rates as the modelled quantity. This family includes different degrees of overlap between populations in the models and parameters for their time-varying index, providing for varying degrees of coherence in the final forecast.

\subsection{Frontier Models}

As well as attempting to jointly model mortality across countries, one can attempt to specify a model that describes how the mortality frontier evolves, and describe how far behind this frontier each individual country is. Bijak et al. (2007) provide population forecasts for 27 European countries using a mortality model based on the assumption that frontier life expectancy increases linearly, and that individual countries converge exponentially toward the frontier with different rates of convergence for males and females. Similarly, Torri and Vaupel (2012) model both frontier life expectancy and the gap between such life expectancy and that of individual countries. The gap is modelled using a logarithm transform to ensure countries always remain below the frontier, and various time-series models are applied to the gap, including the discrete geometric Brownian motion and the discrete geometric mean-reverting process. Pascariu et al. (2018) present a 'two-gap' mortality model, which considers both the gap between the female frontier life expectancy and the equivalent value for any particular country, and the gap between female and male life expectancy in that country, allowing for coherence both between and within countries. Bergeron-Boucher et al. (2018) are concerned with the gap between male and female mortality, and provide a model that constructs a forecast of female mortality, and then separately forecasts male-female mortality ratios. These papers provide ample evidence of the potential efficacy of thinking about mortality forecasting in terms of a mortality frontier. The model presented in this article differs from these approaches in that it attempts to estimates a smooth frontier mortality profile at the level of age-specific rates, based on all available data, and jointly estimates positive deviations from this frontier in a Bayesian hierarchical framework.

\section{Model Specification}

The model presented in this article employs Generalised Additive Models (GAMs) (Wood 2006) to capture both the frontier mortality surface and deviations from it. GAMs model target quantities as sums of smooth functions of covariates, with identifying constraints ensuring such smooths are distinguishable. Hilton et al. (2019) describe a model for mortality forecasting using GAMs. The logarithm of mortality rates are considered as a smooth function of age and cohort, together with smooth age-specific improvement factors 
and non-smoothed period effects. Smooth terms are modelled using penalised B-splines (Wood 2006). The model proposed in this article extends this approach to provide for the inclusion of a mortality frontier. For the sake of simplicity, cohort effects included in the model of Hilton et al. (2019) are jettisoned in order to simplify the development of the model, and an extension of the model could allow their re-inclusion.

Starting from the likelihood, age-specific death counts $D_{x t}$ are given a negative binomial distribution, with a parameter $\exp (\phi)$ determining the degree of over-dispersion relative to the Poisson:

$$
D_{x t} \sim \text { Negative Binomial }\left(m_{x t} R_{x t}, \exp (\phi)\right) .
$$

The $\log$ mortality rate $\log \left(m_{x t}\right)$ is then modelled as a sum of frontier mortality term $f(x, t)$, a country specific term $g^{+}(x, t, c)$ that is constrained to be positive (ensuring, for the most part, that all country rates lie above the frontier), and a period effect $\kappa_{t c}$. For the frontier term, smooth functions of age are used to capture the overall pattern of frontier log-mortality $s_{\beta}(x)$ and the age-specific pattern of mortality improvement factors $s_{\beta}(x)$, assuming that frontier mortality declines linearly. This assumption seems reasonable given the evidence presented in Figure 2, although the distinction between the empirical and modelled frontier should be stressed (the latter aims to discount random variability as well as incorporating assumptions about constant rates of improvement). The country-specific term is considered to be a product of a smooth positive term $s_{\gamma}^{c}(x)$ describing age-specific deviations from the frontier, and an additional term $\exp (h(x, t, c))$ which describes changes in this deviation over time. The exponent in this factor ensures that the overall country-specific term remains positive

$$
\begin{gathered}
\log \left(m_{x t c}\right)=f(x, t)+g^{+}(x, t, c)+\kappa_{t c} \\
f(x, t)=s_{\mu}(x)+s_{\beta}(x) t \\
g^{+}(x, t, c)=s_{\gamma}^{c}(x) \exp (h(x, t, c)) .
\end{gathered}
$$

The function $h(x, t, c)$ describing changes at the level of individual countries can potentially take a number of different forms. As a starting point, we consider $h(x, t, c)$ to comprise a single smooth age term interacting with time $h(x, t, c)=s_{\delta}^{c}(x) t$. Thus, the term $s_{\gamma}^{c}(x)$ can be interpreted as the level of deviation from the frontier at time $t=0$, and the $s_{\delta}^{c}(x)$ term controls the rate of decline or increase of this deviation. The pace of change with respect to time slows as the term $g^{+}(x, t, c)$ tends to zero, so that country-specific rates approach the frontier only asymptotically. However, this model assumes that particular agespecific mortality rates either converge to or diverge from the frontier for particular countries; the direction of change cannot reverse. The introduction of a quadratic term $s_{\lambda}^{c}(x) t^{2}$ rectifies this problem, so that $h(x, t, c)=s_{\delta}^{c}(x) t+s_{\lambda}^{c}(x) t^{2}$.

More varied patterns of deviations from the frontier can be considered by allowing more flexibility in the specification of $h()$. Any number of combinations of age, period and even cohort terms may be included, as long as these are sufficiently constrained so that the other terms in the model are identifiable. Two particular special cases may be important. Firstly, we might allow for variations in the pace and direction of mortality change by incorporating the bi-variate form of Lee and Carter (1992), so that $h(x, t, c)=s_{\delta}^{c}(x) k_{\mathrm{tc}}$. In this case, we would no longer include the period term $\kappa_{t c}$, as its function would be subsumed by the new $k_{t c}$ term. The usual Lee-Carter constraints would be required to ensure identifiability. 
Secondly, an even greater degree of flexibility might be provided by including a twodimensional spline term $h(x, t, c)=s_{\eta}^{c}(x, t)$, in the spirit of the model of Currie et al. (2004). Again, constraints would be required in order to identify such effects. Furthermore, the introduction of bivariate terms complicates matters both conceptually and computationally. Preliminary experiments encountered difficulties in estimating these models, although we do not believe these are insurmountable. For this article, the simpler parametric forms are retained, although future work may benefit from investigating this link.

All smooth terms are modelled using penalised B-splines (Wood 2006). Separate Bspline basis functions of age are defined for the frontier mortality term and the countryspecific deviations, allowing a larger number of knots to be used to capture the pattern of frontier mortality:

$$
\begin{aligned}
& s_{\mu}(x)=B_{f}(x) \boldsymbol{\mu} \\
& s_{\beta}(x)=B_{f}(x) \boldsymbol{\beta} \\
& s_{\gamma}^{(c)}(x)=B_{g}(x) \boldsymbol{\gamma}_{\mathbf{c}} \\
& s_{\delta}^{(c)}(x)=B_{g}(x) \boldsymbol{\delta}_{c} \\
& s_{\lambda}^{(c)}(x)=B_{g}(x) \boldsymbol{\lambda}_{c} \\
& s_{\eta}^{c}(x, t)=\left(B_{g}(x) \otimes B_{l}(t)\right) \eta_{c} .
\end{aligned}
$$

First difference prior penalties are applied to basis function coefficients to ensure smoothness with respect to age and to guard against over-fitting (Wood 2006; Lang and Brezger 2004). As in Hilton et al. (2019), the null space of these penalties is penalised separately to ensure that the resulting prior is proper. The matrix of country-specific basis function coefficients $\Gamma=\left(\boldsymbol{\gamma}_{1}, \boldsymbol{\gamma}_{2}, \ldots, \gamma_{C}\right)$, which determine the main deviation term $s_{\gamma}(x)$, is treated slightly differently. These coefficients are constrained to be positive, ensuring that the smooth term as a whole is positive everywhere, as all elements of the matrix of basis functions $B(\boldsymbol{x})$ are positive. As with other terms, the coefficient matrix has a smoothness prior applied to each column penalising first differences in the age direction (Currie et al. 2004), but also double exponential random effect priors applied across each row, with separate variance parameters. The later prior pulls country-specific deviations toward zero, in effect ensuring that the frontier remains close to the lowest observed mortality rates at each age. The full prior specification for $\Gamma$ is:

$$
\begin{array}{r}
\gamma_{y}=\left(\gamma_{y 1}, \gamma_{y 2}, \ldots, \gamma_{y C}\right)^{T} \\
\gamma_{y c}>0 \text { for all } y, c \\
\gamma_{y c} \sim N\left(0, \sigma_{y}\right) \\
\sigma_{y} \sim \operatorname{Exponential}(0.2),
\end{array}
$$

where $y$ indexes a particular basis function in $B_{\mathrm{g}}(x)$. 
The period effect $\kappa_{t c}$ is a country-specific random walk capturing year-to-year random variation in mortality caused by factors such as flu and temperature variations. In order to ensure that the overall time-trends are captured in the other model parameters, the $\kappa$ term is constrained so that it sums to zero, and contains no linear or quadratic components. The random walk prior is adjusted to account for these constraints in a similar way to Hilton et al. (2019). One limitation introduced by specifying the period effect in this way is that it makes it possible for individual countries to dip below the frontier in the short term. This problem is mitigated to some extent by the constraints on the period term; these prevent the country rates from straying systematically below the frontier. Thus, where rates do fall below the frontier, these indicate short-term aleatory deviations rather than a sustained trend, and do not undermine the structure of the model. In the examples that follow, period effects of different countries are considered independent, although the prior correlation structure could be specified in greater detail, allowing different levels of correlation between countries, or accounting for geographical or social-cultural factors that might induce correlation between mortality rates across countries.

In summary, the proposed model has some desirable features. Firstly, it produces smooth estimates and forecasts of mortality with associated uncertainty. Secondly, although mortality improvements in particular countries may wane and wax in the short term (Case and Deaton 2017), the overall global decline in best-practice mortality appears to be relatively consistent.

This model provides a means of estimating a smooth profile and rate of change for this frontier mortality. Thirdly, where a particular country has displayed fast decline in mortality, we anticipate that this growth will slow as that country approaches the limits of what is currently possible. This model formalises this assumption by ensuring country mortality is limited by the level of the frontier.

\section{Data and Estimation}

The Human Mortality Database (Human Mortality Database 2019) was used to obtain agespecific death and exposure data for 19 developed countries with reasonably large populations and for which data is available for at least the period 1961 and onward. Only female data are used in this instance; future work could plausibly consider modelling males jointly by extending the 'double-gap' life-expectancy model of Pascariu et al. (2018) to a mortality rate context. Infant mortality and centenarians were excluded, although extending the model to incorporate these age groups should be possible. Data from 1961-2006 is used to fit the three models: the linear and quadratic variants of the proposed model and comparator model where each country is fitted independently. Data from 2007-2016 held back for purposes of assessment. Table 1 provides a list of the countries used.

The frontier and country-specific elements of the models were fitted jointly using the stan Bayesian modelling software (Stan Development Team 2019). Each model run consisted of four chains, each consisting of 8000 iterations, with the first half of each chain used to optimise the relevant sampling parameters and discarded, and additionally the remaining samples were thinned by a factor of two, to reduce memory usage. Diagnostic measures suggested that each chain had converged to the target distribution. The four chains were run in parallel, with sampling taking 37 hours for the frontier model results presented here. 
Table 1. List of country data from the Human Mortality Database used in model estimation.

\begin{tabular}{l}
\hline Country name \\
\hline Australia \\
Austria \\
Belgium \\
Denmark \\
Finland \\
France \\
West Germany \\
Spain \\
Ireland \\
Japan \\
Netherlands \\
New Zealand (non-Maori) \\
Norway \\
Portugal \\
Sweden \\
Switzerland \\
England and Wales \\
Scotland \\
USA
\end{tabular}

\section{Results}

\subsection{Frontier Posterior}

In this section, model results are presented for the quadratic model variant. Starting with the frontier model, Figure 5 shows the posterior distribution of the frontier surface defined by $s_{\mu}(x)+s_{\beta}(x) t$ at selected years. These distributions are plotted together with corresponding empirical log rates for the 19 countries included in the estimation processes. Each country is displayed in a different colour, although distinguishing individual countries, observations is not important for the interpretation of the chart. The frontier estimates lie below, but close to, the vast majority of observed rates. At younger ages, some, observations lie beyond the frontier. This is to be expected, as the estimated frontier is supposed to represent the lower limit of the central rate $m_{x, t}$, but it does not account for the additional negative binomial uncertainty in deaths. In other words, although the force of mortality will generally lie above the frontier, random variation in realised death counts could result in observed rates that lie below it. Thus, the empirical mortality frontier is distinct from the 'true' mortality frontier that we are trying to model. Younger ages are more likely to display this effect, because mortality is much lower at these ages, and so the effect of negative binomial uncertainty on observed log-rates is far greater.

It should also be noted that unlike the country-specific deviations, the period effect for particular years $\kappa_{t c}$ may be negative, and in some cases this may result in modelled mortality rates that lie below the frontier. Given that the scale of the period effects is generally small relative to the deviations, this will only occur for countries that are already very close to the frontier, and is not deemed to be a significant shortcoming in the model specification. 
The final panel in Figure 5 is a forecast for 2016. Again, observations for the majority of the age range appear consistent with our interpretation of the frontier, although it is possible that decline in the frontier for young adults aged 20-30 is slightly underestimated by the model.

Moving on to the results for individual countries, Figure 6 displays the posterior of country-specific deviation term $s_{\gamma}^{c}(x)$ by age for a few selected countries, namely France, England and Wales, Japan, and Norway. Results for all countries are provided in the supplementary materials. This term defines deviations at the intercept of the time index

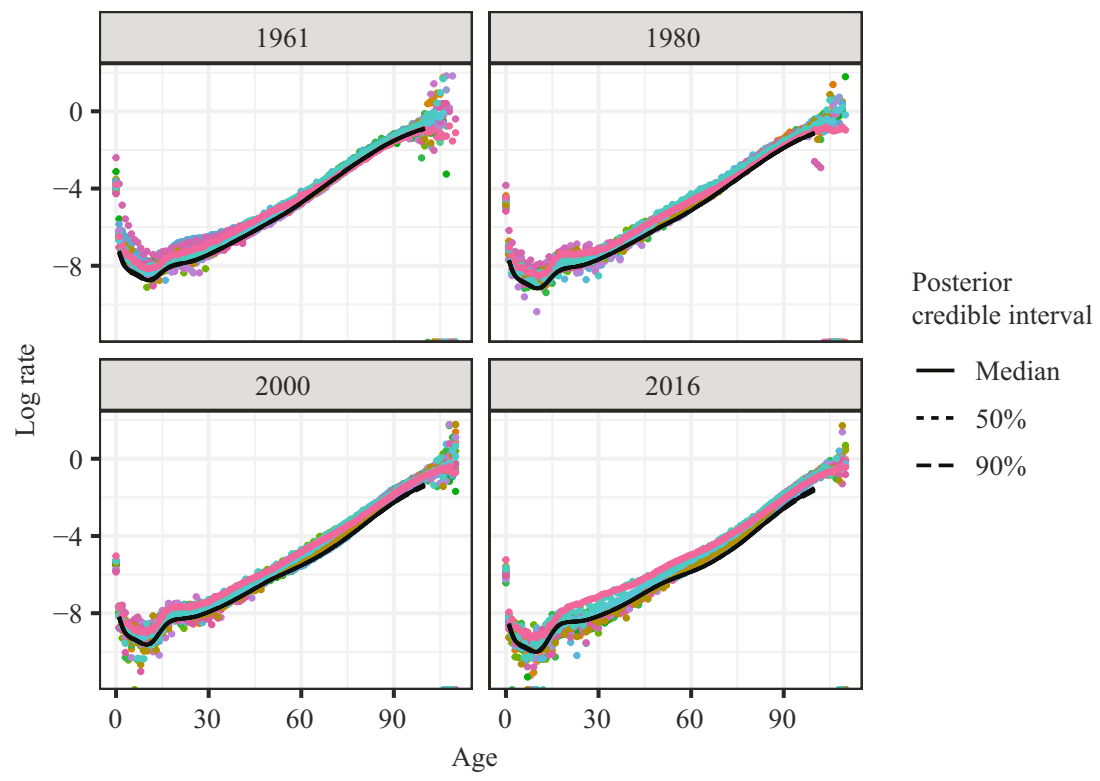

Fig. 5. Posterior distribution of frontier mortality, selected years. Plotted data points represent all observations in a given year; colours denote countries.
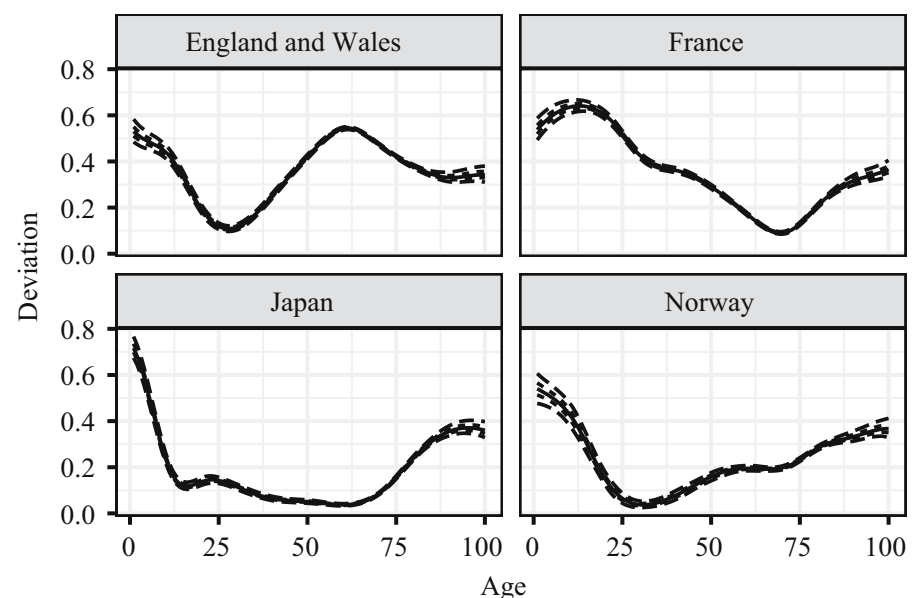

Posterior

credible interval

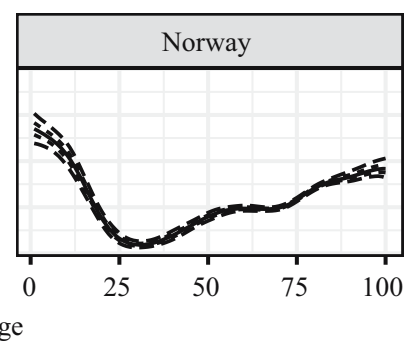

- Median

-- $50 \%$

- - $90 \%$

Fig. 6. Posterior distribution of country-specific deviations at the intercept of the time index for selected countries. 
variable $t$. For fitting, this index is centred and normalised, so the deviations displayed correspond to the distance from the frontier in the middle of the fitting period 1961-2006 (about 1983). One can see that England and Wales approach best-practice mortality for young adults, but are further away at age 60 . In contrast, Japanese mortality is very close to frontier from ages 30-60, while French mortality appears to take the lead around age 60 .

Knowledge of the extent of deviations may provide useful information for government bodies and service providers. If a particular country appears to be lagging behind in bestpractice mortality at particular age groups, this may provide a useful target for future interventions. Comparing the speed of convergence towards the frontier with similar countries may also provide useful benchmarking information.

A key question is how effectively the model can fit observed data and predict future trends in mortality. For illustrative purposes, we display posterior distributions for particular agespecific rates across time for England and Wales in Figure 7. Empirical rates are plotted as red dots, while the beginning of the forecast period is indicated by a black horizontal line. The posterior mean for each age-specific rate lies above frontier mortality boundary. Most empirical observations lie within the $90 \%$ credible interval, both over the fitting period and for the forecasts, indicating that the model does a reasonable job at capturing our uncertainty

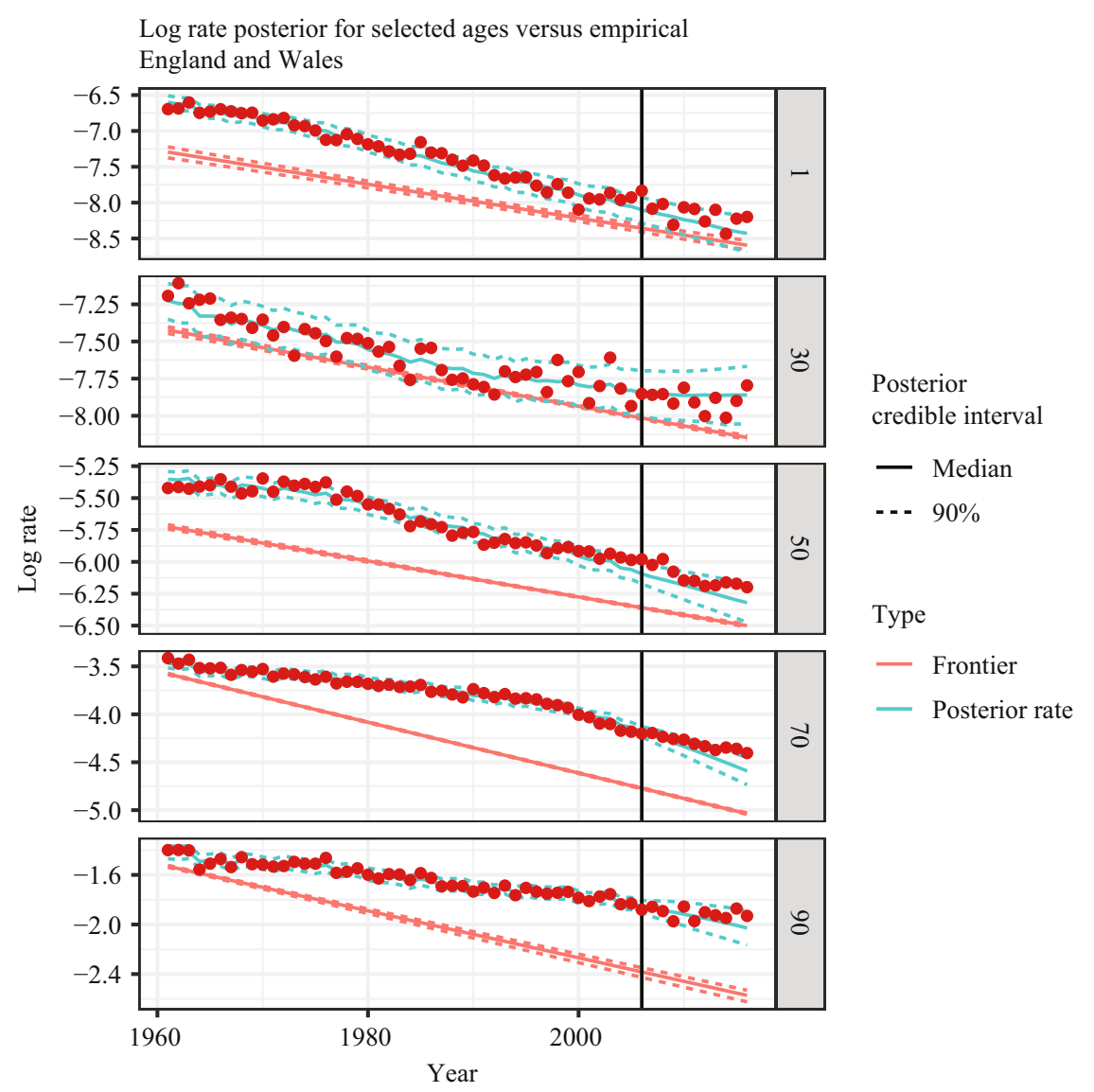

Fig. 7. Posterior predictive distribution of log-mortality rates for selected ages, England and Wales. 
about the data. There is some evidence that our forecasts are overly optimistic about the extent to which mortality for England and Wales will decline towards the frontier around age 70; here the last few observations fall outside the predictive interval.

Of course, a more thorough examination of the model is needed to decide its efficacy. Extensive plots for all countries can be found in the supplemental material. It is evident that for the quadratic model in particular, some countries display unrealistic forecasts at particular ages; the cause of and potential remedy for this issue is discussed in Section 8. For the purposes of formal assessment, root-mean squared error (RMSE) and empirical coverage (the proportion of observations falling within the posterior interval of a given probability) were calculated over the forecast period 2007-2016 for all countries. RMSE was calculated using the mean of the posterior rate for each forecast year and age as the relevant point estimates. One goal of the assessment is to provide evidence that including information about the frontier is useful for forecasting. To this end, a series of models were fitted to each country independently that included only smooth age, age-specific improvement, and period terms:

$$
\log \left(m_{x t}\right)=s_{\mu}(x)+s_{\beta}(x) t+\kappa_{t} .
$$

Thus, we can compare the forecast performance of the model in which country forecasts are independent (labelled 'Independent' in subsequent plots) with variants of the frontier model we are proposing. Specifically, we investigate two different choices of the $h(x, t, c)$ function determining the change in country mortality relative to the frontier:

$$
\begin{gathered}
h_{1}(x, t, c)=s_{\delta}(x) t \\
h_{2}(x, t, c)=s_{\delta}(x) t+s_{\lambda}(x) t^{2} .
\end{gathered}
$$

These are referred to as the linear and quadratic models respectively. To give a clear idea of whether these variants are doing better than the comparator independence model, Figure 8 displays the difference between RMSE for the variants and the independence models for each

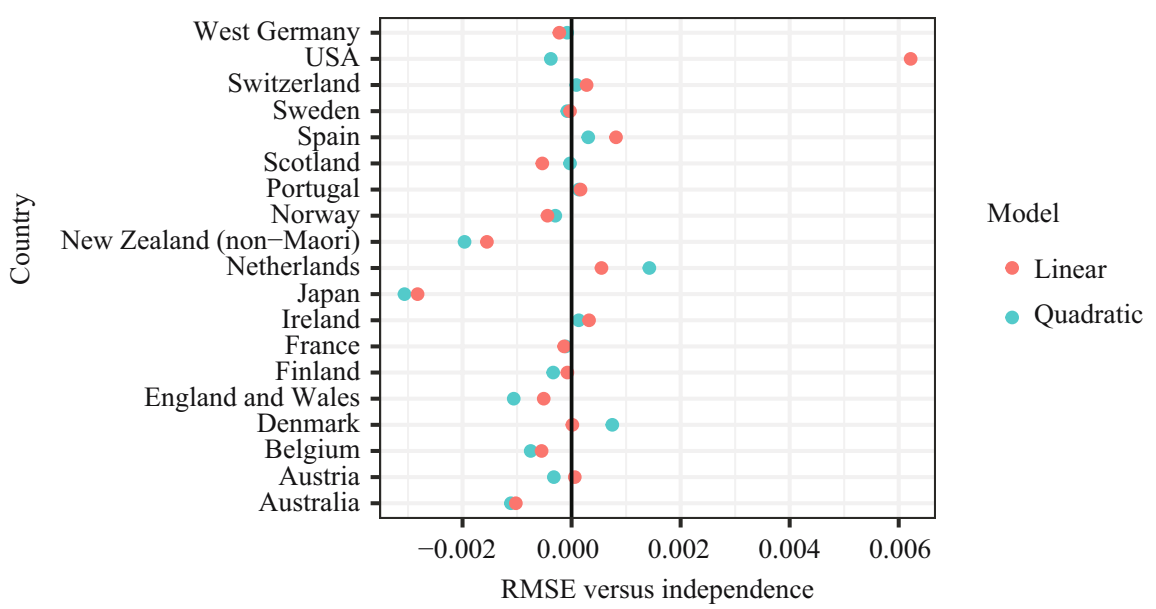

Fig. 8. Difference between RMSE of frontier model variants and a similar model fitted independently.

Note: The data here does indeed refer to (the geographical region of) the old West Germany. The Human Mortality Database maintains a separate time series for this region. It is used here because it allows the use of a consistent series going back to 1946, which allows more reliable estimates than would be possible if only data for the unified country from 1990 onwards was used. 


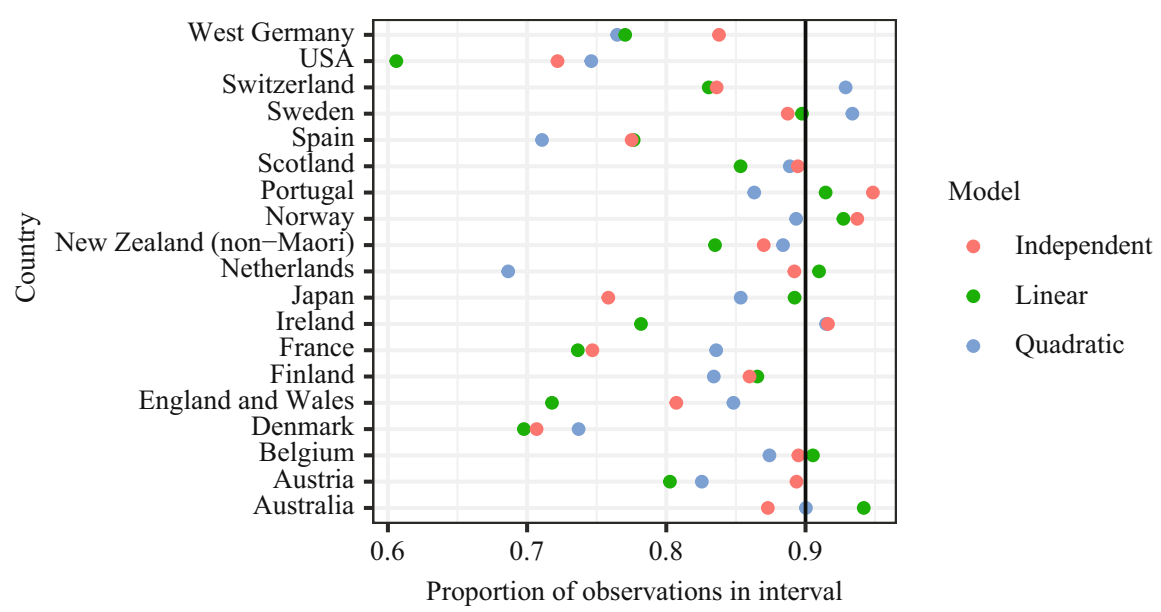

Fig. 9. Proportion of observations falling within $90 \%$ predictive interval for the independent model, and linear and quadratic variants of the frontier model.

Note: The data here does indeed refer to (the geographical region of) the old West Germany. The Human Mortality Database maintains a separate time series for this region. It is used here because it allows the use of a consistent series going back to 1946, which allows more reliable estimates than would be possible if only data for the unified country from 1990 onwards was used.

country. If this value is negative (to the left of the axis at zero in the chart), it indicates that the variant model performs better. If it is positive, the reverse is true. The assessment reveals that for 13 of the 19 countries, the quadratic model has a lower RMSE over the forecast period than the independent model. For the linear model, the results are closer: it is preferred by this metric over the independence model in 11 of 19 cases.

The accuracy of point estimates are not the only relevant area of assessment. Quantification of uncertainty in forecasts is important in managing longevity risk, and so the extent to which observations fall within forecast intervals is also important. Figure 9 provides the proportion of observations that fall within the central $90 \%$ predictive probability interval. Ideally, this value should approach $90 \%$, indicating that forecast uncertainty appears well calibrated. However, given that for each country we only observe one correlated set of rates (over the period 2006-2016), this proportion does not correspond exactly with the frequentist interpretation of coverage, which relies on independent replications of the same experiment. Therefore, we must not over-interpret the reported empirical coverage statistics. In general, the results are encouraging. A majority of all models have empirical coverages ranging between $80 \%$ and $95 \%$ for the $90 \%$ interval. The quadratic model has five observations with coverages below $80 \%$, compared to five for the independent model and seven for the linear variant. The United States, Denmark and Spain appear to have patterns of recent mortality decline that are difficult to capture for all models. The quadratic model appears to be the better performing model overall based on these metrics, although it appears to perform particularly poorly for both RMSE and coverage in the case of the Netherlands.

\section{Discussion}

This article has set out a model of mortality that estimates the evolution of frontier mortality as a set of smooth rates, and then considers individual countries as deviations 
from this profile. Frontier mortality is constrained to lie below the modelled force of mortality for all individual countries, but the prior specification ensures that it remains close to best-performing countries by penalising the magnitude of the individual country deviations. Estimates of frontier mortality and the extent of particular country deviations from this standard may provide useful benchmarking information to public bodies. The model was fitted jointly to 19 countries, and its performance in short-term forecasting is compared to a similar model without a frontier component, in which each country was modelled independently. The frontier model was found to perform somewhat better in terms of the accuracy of its central forecasts than the independence model over a ten-year time horizon. These findings suggest that a frontier model has potential for use in forecasting mortality for a large group of countries, perhaps particularly by multinational bodies with access to harmonised data from a variety of sources.

Some limitations and areas for future investigation can be identified. Firstly, a longer time horizon may be required to accurately assess the usefulness of the model. Mortality forecasts are typically used to compute cohort life expectancies, which require considerable longer forecasts than have been provided here. Secondly, forecasts for females only were produced in the examples above. Extending the approach described to multiple sexes using a 'double-gap' model, as employed by Pascariu et al. (2018) for life expectancy, may have some utility. Thirdly, at present simple linear and quadratic terms were chosen to describe the evolution of country specific deviations from the frontier. These may not be the best choices for this element of the model. In particular, over longer time horizons, the quadratic model may predict unrealistic divergences from the frontier at some ages in countries where recent stagnation in mortality rates have been observed, leading in some cases to predicted increases in mortality. Section 5 sets out two possible alternative models based on Lee and Carter (1992) and Currie et al. (2004) that require further investigation. Specifying priors on the time-varying elements of these models that favour mean-reversion will help to ensure forecast means do not diverge from the frontier over the long term. Finally, a comparison between frontier models and those that provide for convergence towards a mean trend might be investigated; it may be that such models produce similar conclusions, or that one or another is more efficacious.

\section{References}

Aburto, J.M., F. Villavicencio, U. Basellini, S. Kjærgaard, and J.W. Vaupel. 2020. "Dynamics of life expectancy and life span equality." Proceedings of the National Academy of Sciences of the United States of America 117 (10): 5250-9. DOI: https:// doi.org/10.1073/pnas.1915884117.

Alho, J.M. 2019. "Forecasting Life Expectancy: A Statistical Look at Model Choice and Use of Auxiliary Series." In Old and New Perspectives on Mortality Forecasting, edited by Tommy Bengtsson and Nico Keilman, 185-95. Springer Open. DOI: https://doi.org/ 10.1007/978-3-030-05075-7_15.

Bengtsson, T. 2019. "Linear Increase in Life Expectancy: Past and Present." In Old and New Perspectives on Mortality Forecasting, edited by Tommy Bengtsson and Nico Keilman, 221 -34. Springer Open. DOI: https://doi.org/10.1007/978-3-030-05075-7_17. 
Bergeron-Boucher, M.P., V. Canudas-Romo, J. Oeppen, and J.W. Vaupel. 2017. "Coherent forecasts of mortality with compositional data analysis." Demographic Research 37 (1): 527-66. DOI: https://doi.org/10.4054/DemRes.2017.37.17.

Bergeron-Boucher, M.P., V. Canudas-Romo, M. Pascariu, and R. Lindahl-Jacobsen. 2018. "Modeling and forecasting sex differences in mortality: a sex-ratio approach." Genus 74 (1). DOI: https://doi.org/10.1186/s41118-018-0044-8.

Biatat, V.D., and I.D. Currie. 2010. "Joint models for classification and comparison of mortality in different countries." In Proceedings of 25rd International Workshop on Statistical Modelling: 89-94. 5-9 July 2010, Glasgow, UK. Available at: http:// www.statmod.org/workshops_archive_proceedings_2010.htm (accessed October 2020).

Bijak, J., D. Kupiszewska, M. Kupiszewski, K. Saczuk, and A. Kicinger. 2007. "Population and labour force projections for 27 European countries, 2002-2052: Impact of international migration on population ageing." European Journal of Population 23 (1): 1-31. DOI: https://doi.org/10.1007/s10680-006-9110-6.

Bohk-Ewald, C., and R. Rau. 2017. "Probabilistic mortality forecasting with varying agespecific survival improvements.” Genus 73 (1): 1-37. DOI: https://doi.org/10.1186/ s41118-016-0017-8.

Booth, H., R. Hyndman, L. Tickle, and P. de Jong. 2006. "Lee-Carter mortality forecasting: a multi-country comparison of variants and extensions." Demographic Research 15 (9): 289-310. DOI: https://doi.org/10.4054/DemRes.2006.15.9.

Booth, H., and L. Tickle. 2008. "Mortality Modelling and Forecasting: A Review of Methods." Annals of Acturial Science 3 (1-2): 3-43. DOI: https://doi.org/10.1017/ S1748499500000440.

Cairns, A.J.G., D. Blake, K. Dowd, G.D. Coughlan, D. Epstein, A. Ong, I. Balevich, D. Coughlan, D. Epstein, and A. Ong. 2009. “A Quantitative Comparison of Stochastic Mortality Models Using Data from England and Wales and the United States." North American Actuarial Journal 13 (March): 1-35. DOI: https://doi.org/10.1080/ 10920277.2009.10597538.

Cairns, A.J.G., D. Blake, K. Dowd, G.D. Coughlan, and M. Khalaf-Allah. 2011. "Bayesian stochastic mortality modelling for two populations.” ASTIN Bulletin 41 (1): 29-59. DOI: https://doi.org/10.2143/AST.41.L2084385.

Case, A., and A. Deaton. 2017. "Mortality and morbidity in the 21st century." Brookings Papers on Economic Activity 2017 (Spring): 397-476. DOI: https://doi.org/10.1353/ eca.2017.0005.

Currie, I.D., M. Durban, and P.H.C. Eilers. 2004. "Smoothing and forecasting mortality rates." Statistical Modelling 4: 279-98. DOI: https://doi.org/10.1191/1471082x04st 080oa.

Enchev, V., T. Kleinow, and A.J.G. Cairns. 2017. "Multi-population mortality models: fitting, forecasting and comparisons." Scandinavian Actuarial Journal 2017 (4): 319-42. DOI: https://doi.org/10.1080/03461238.2015.1133450.

Hilton, J., E. Dodd, J. Forster, and P.W.F. Smith. 2019. "Projecting UK Mortality using Bayesian Generalised Additive Models." Journal of the Royal Statistical Society. Series C 68 (1): 29-49. DOI: https://doi.org/10.1111/rssc.12299. 
Human Mortality Database. 2019. "Human Mortality Database.” University of California, Berkeley (USA); Max Planck Institute for Demographic Research (Germany). Available at: DOI: http://www.mortality.org/cgi-bin/hmd. (accessed in October 2019). Hyndman, R.J., H. Booth, and F. Yasmeen. 2013. "Coherent Mortality Forecasting: The Product-Ratio Method with Functional Time Series Models." Demography 50 (1): 261-83. DOI: https://doi.org/10.1007/s13524-012-0145-5.

Hyndman, R.J., and Md. Shahid Ullah. 2007. "Robust forecasting of mortality and fertility rates: A functional data approach.” Computational Statistics and Data Analysis 51 (10): 4942-56. DOI: https://doi.org/10.1016/jxsda.2006.07.028.

Keyfitz, N. 1977. "What difference would it make if cancer were eradicated? An examination of the taeuber paradox." Demography 14 (4): 411-18. DOI: https://doi. org/10.2307/2060587.

Kleinow, T. 2015. "A common age effect model for the mortality of multiple populations." Insurance: Mathematics and Economics 63. Elsevier B.V.: 147-52. DOI: https://doi. org/10.1016/j.insmatheco.2015.03.023.

Lang, S., and A. Brezger. 2004. "Bayesian P-Splines." Journal of Computational and Graphical Statistics 13 (1): 183-212. DOI: https://doi.org/10.1198/1061860043010.

Lee, R.D., and S. Tuljapurkar. 1994. "Stochastic population forecasts for the United States: beyond high, medium, and low." Journal of the American Statistical Association 89 (428): 1, 1175-89. DOI: http://www.jstor.org/stable/10.2307/2290980.

Lee, R. 2019. "Mortality Forecasts and Linear Life Expectancy Trends." In Old and New Perspectives on Mortality Forecasting, edited by Tommy Bengtsson and Nico Keilman, 167-83. Springer Open. DOI: https://doi.org/10.1007/978-3-030-05075-7_14.

Lee, R.D, and L.R. Carter. 1992. "Modeling and Forecasting U.S Mortality." Journal of the American Statistical Association 87 (419): 659-71. Available at: http://www.jstor. org/stable/2290201.

Li, N., and R. Lee. 2005. "Coherent mortality forecasts for a group of populations: An extension of the Lee-Carter method." Demography 42 (3): 575-94. DOI: https://doi. org/10.1353/dem.2005.0021.

Li, N., R. Lee, and P. Gerland. 2013. "Extending the Lee-Carter method to model the rotation of age patterns of mortality decline for long-term projection." Demography 50 (6): 2037-51. DOI: https://doi.org/10.1007/s13524-013-0232-2.

Luy, M. 2003. "Causes of Male Excess Mortality: Insights from Cloistered Populations." Population and Development Review 29 (4). Blackwell Publishing Ltd.: 647-76. DOI: doi.org/10.1111/j.1728-4457.2003.00647.x.

Oeppen, J. 2019. "Life Expectancy Convergence Among Nations Since 1820: Separating the Effects of Technology and Income." In Old and New Perspectives on Mortality Forecasting, edited by Tommy Bengtsson and Nico Keilman, 197-219. Springer Open. DOI: https://doi.org/10.1007/978-3-030-05075-7_16.

Oeppen, J., and J.W. Vaupel. 2002. "Broken Limits to Life Expectancy." Science 296 (5570): 1029-31. DOI: https://doi.org/10.1126/science.1069675.

Olshansky, S.J., B.A. Carnes, and A. Desesquelles. 2001. "Prospects for Human Longevity." Science 291 (5508): 1492-2. DOI: https://doi.org/10.1126/science.291. 5508.1491. 
Pascariu, M.D., V. Canudas-Romo, and J.W. Vaupel. 2018. "The doublegap life expectancy forecasting model." Insurance: Mathematics and Economics 78 (2018): 339-50. DOI: https://doi.org/10.1016/j.insmatheco.2017.09.011.

Raftery, A.E., J.L. Chunn, P. Gerland, and H. Sevcikova. 2013. "Bayesian Probabilistic Projections of Life Expectancy for All Countries.” Demography 50 (3): 777-801. DOI: https://doi.org/10.1007/s13524-012-0193-X.

Schinzinger, E., M.M. Denuit, and M.C. Christiansen. 2016. "A multivariate evolutionary credibility model for mortality improvement rates." Insurance: Mathematics and Economics 69: 70-81. DOI: https://doi.org/10.1016Zj.insmatheco.2016.04.004.

Stan Development Team. 2019. "Stan Modeling Language Users Guide and Reference Manual." Available at: DOI: http://mc-stan.org/index.html (accessed in October 2019).

Torri, T., and J.W. Vaupel. 2012. "Forecasting life expectancy in an international context." International Journal of Forecasting 28 (2): 519-31. DOI: https://doi.org/10.1016/j. ijforecast.2011.01.009.

Tuljapurkar, S., N. Li, and C. Boe. 2000. "A universal pattern of mortality decline in the G7 countries.” Nature 405 (6788): 789-92. DOI: https://doi.org/10.1038/35015561.

Vallin, J., and F. Mesle. 2009. "The segmented trend line of highest life expectancies." Population and Development Review 35 (1): 159-87. DOI: https://doi.org/10.1111/ j.1728-457.2009.00264.x.

Vaupel, J.W. 1986. "How Change in Age-Specific Mortality Affects Life Expectancy." Population Studies 40 (1): 147-57. DOI: https://doi.org/10.1080/0032472031000141896.

Vaupel, J.W., and V. Canudas Romo. 2003. "Decomposing change in life expectancy: A bouquet of formulas in honor of Nathan Keyfitz's 90th birthday.” Demography 40 (2): 201-16. DOI: https://doi.org/10.2307/3180798.

Vaupel, J.W., and V. Canudas Romo. 2000. "How Mortality Improvement Increases Population Growth.” In Optimization, Dynamics, and Economic Analysis: Essays in Honour of Gustav Feichtinger, edited by Engelbert J Dockner, Richard F Hartl, Mikulas Luptacik, and Gerhard Sorger, 345-52. Berlin/Heidelberg: Springer-Verlag. DOI: https://doi.org/10.1007/978-3-642-57684-3_29.

Wachter, K.W. 1997. "Between Zeus and the Salmon: Introduction." In Between Zeus and the Salmon, edited by Kenneth W Wachter and Caleb E Finch. Washington DC: National Academy Press.

Wachter, K.W., D. Steinsaltz, and S.N. Evans. 2014. "Evolutionary shaping of demographic schedules." Proceedings of the National Academy of Sciences of the United States of America 111 (SUPPL.3): 10846-53. DOI: https://doi.org/10.1073/ pnas. 1400841111.

Wilson, C. 2001. "On the scale of global demographic convergence, 1950-2000." Population and Development Review 27 (1): 155-71. DOI: https://doi.org/10.1111/j. 1728-4457.2001.00155.x.

Wood, S.N. 2006. Generalised Additive Models: An Introduction with R. Boca Raton: Chapman; Hall / CRC Press.

Received November 2019

Revised July 2020

Accepted November 2020 